\title{
Giant growth rate in nano-oxidation of $p$-silicon surfaces by using ethyl alcohol liquid bridges
}

\author{
Marta Tello and Ricardo García ${ }^{\text {a) }}$ \\ Instituto de Microelectrónica de Madrid, CSIC, Isaac Newton 8, 28760 Tres Cantos, Madrid, Spain
}

(Received 2 May 2003; accepted 28 July 2003)

\begin{abstract}
We demonstrate that local oxidation nanolithography can be performed in liquid environments different from aqueous solutions with a significant improvement in the aspect ratio of the fabricated motives. Here, we perform a comparative study of noncontact atomic force microscopy oxidation experiments in water and ethyl alcohol. The growth rate of local oxides can be increased by almost an order of magnitude by using oxyanions from ethyl alcohol molecules. We propose that the enhanced growth rate is a consequence of the reduction of the trapped charges within the growing oxide. The present results open the possibility of using local oxidation nanolithography to directly fabricate vertical oxide structures while keeping lateral sizes in the nanometer range. (C) 2003 American Institute of Physics. [DOI: 10.1063/1.1613799]
\end{abstract}

Local oxidation or nano-oxidation of semiconductor and metallic surfaces by atomic force microscopy (AFM) is emerging as a reliable and versatile lithographic method for the fabrication of nanometer-scale structures and devices. ${ }^{1-9}$ The present knowledge allows establishing some similarities to conventional anodic oxidation. The AFM tip is used as a cathode and the water meniscus formed between tip and surface is the source of the oxyanions species. ${ }^{2,10-15}$ The strong localization of the electrical field lines near the tip apex and the lateral confinement of the oxyanions species within the liquid meniscus gives rise to a nanometer-size oxide dot. The majority of local oxidation experiments have used a water meniscus formed either spontaneously by the mechanical contact between tip and sample or field-induced by the application of an external voltage. ${ }^{2,16}$ Only in two isolated instances other sources of oxyanions were used. Marchi et al. ${ }^{17}$ used ozone enriched environments and Wei and Eres used oxyanions produced by the electron-beam-induced dissociation of residual water vapor in a vacuum chamber. ${ }^{18}$ In both cases, oxidation marks were generated, however, the experimental setups were somehow impractical and have not been pursued.

The local oxidation process in aqueous environments is characterized by a high initial growth rate that decays rapidly and finally saturates. Among the challenges to establish local oxidation as an alternative lithographic method to pattern surfaces is the control of the vertical growth rate. Space charge buildup within the growing oxide, increasing oxide thickness, buildup of stress because oxidation-induced volume expansion, and mechanical tip-oxide forces are the likely factors responsible for the progressive slowing down of the oxidation kinetics. In practical terms this means that local oxide structures are usually in the 5-10 $\mathrm{nm}$ range in thickness or 3-6 $\mathrm{nm}$ in height.

Here, we study the possibility of using different electrolytes to enhance the vertical growth rate of local oxides. Our choice has been to use alcohols, in particular ethyl alcohol $\mathrm{CH}_{3}-\mathrm{CH}_{2} \mathrm{OH}$, because of its dipole moment is comparable

${ }^{a)}$ Electronic mail: rgarcia@imm.cnm.csic.es to that of the water $(1.69$ vs $1.85 \mathrm{D})$, and the potential abundance of oxyanions $\mathrm{OH}^{-}$. Its polar character allows the field-induced formation of alcohol bridges between tip and sample. We have performed a comparative study of the height and width dependencies on voltage pulse conditions by using water and ethyl alcohol bridges, respectively. Besides, we demonstrate that local oxidation can also be performed in nonaqueous liquids.

The experiments were performed with a dynamic atomic force microscope operated in the low amplitude solution (noncontact) ${ }^{19}$ and with additional circuits to perform the oxidation. $^{2}$ The microscope was placed into a closed box with inlets for dry nitrogen and alcohol vapors. First, the chamber was purged of water vapor by flushing dry $\mathrm{N}_{2}$ for about $30 \mathrm{~min}$. This reduced the relative humidity below $2 \%$. Then, the chamber was filled with ethyl alcohol vapor. Noncontact AFM oxidations were performed with doped $n^{+}$-type silicon cantilevers (Nanosensors, Germany). The force constant $k_{c}$ and resonance frequency $f_{0}$ were about $25 \mathrm{~N} / \mathrm{m}$ and $280 \mathrm{kHz}$, respectively. The cantilever was excited at its resonance frequency. The semiconductor samples were $p$-type $\mathrm{Si}(100)$ with a resistivity of $0.1-1.4 \Omega \mathrm{cm}$. The numerical data presented in Figs. 2-3 is the average of five oxidation experiments under the same conditions (the standard deviation is approximately $10 \%$ of the average value). The alcohol meniscii bridging tip and sample were field-induced by applying an external voltage between tip and sample. The protocol is similar to the one previously applied to field-induced the formation of water bridges. ${ }^{2,16}$

Figure 1 shows a typical sequence of oxide dots obtained by bridging tip and sample with ethyl alcohol and water capillaries, respectively. The dots have been obtained by applying a sequence of $20 \mathrm{~V}$ pulses (tip negative) for different times $(0.001,0.003,0.01,0.03,0.1,0.3,1,3,10$, and $30 \mathrm{~s})$. The cross section [Fig. 1(b)] reveals that there is hardly any oxidation growth in ethyl alcohol environments for oxidation times smaller than $0.01 \mathrm{~s}$. However, a dramatic increase in the growth rate and consequently in the height values are observed for longer pulses. The oxidation growth and the height values are considerable smaller in water, Figs. 1(c) and $1(\mathrm{~d})$, although oxides are produced for very short pulses. 


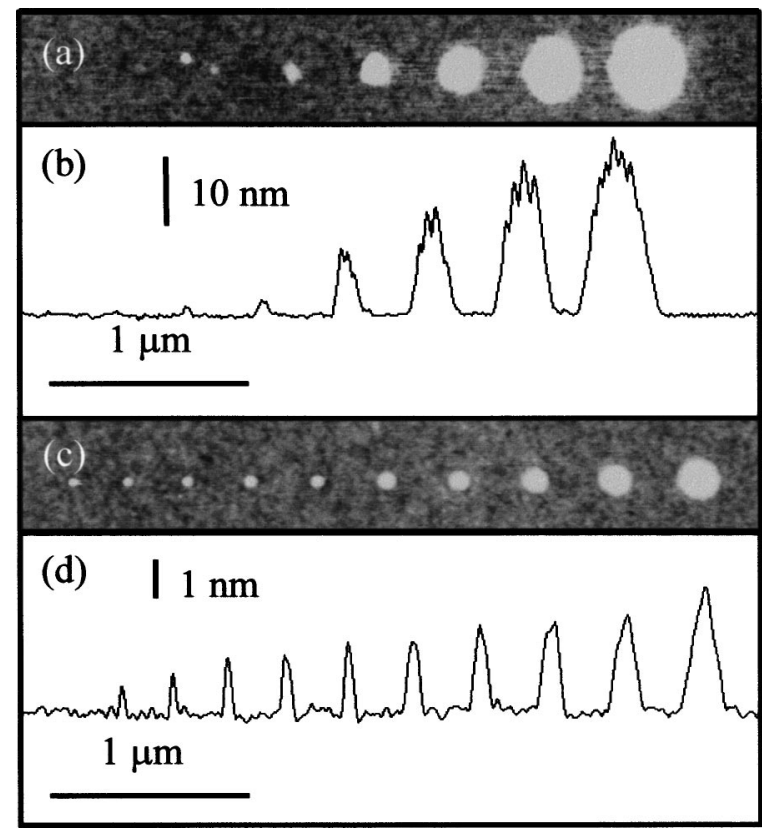

FIG. 1. AFM image of a sequence of oxidation experiments performed for different pulse duration $(0.001,0.003,0.01,0.10,0.3,1,3,10$, and $30 \mathrm{~s}$ from left to right) and constant voltage (20 V): (a) in ethyl alcohol, (b) cross section of (a), (c) in water, and (d) cross-section of (c).

For a fixed voltage and oxidation time there is considerable dispersion in the oxidation heights obtained by using different, although in principle similar tips. We find an standard deviation of about $25 \%$ of the mean value. ${ }^{20}$ To rule out the possibility that either variations in the tip's doping concentration or in its geometry could modify the height values obtained at otherwise identical conditions, we have performed a comparative oxidation experiment where the same tip was used to generate a sequence of dots in aqueous and ethyl alcohol environments. The protocol requires that a single tip performs a sequence of oxidation experiments in water, alcohol, and water. The data presented here only includes sets of experiments where initial and final oxidations in water gave the same numerical values. This protocol eliminates any possible artifact due to tip changes in the interpretation of the oxidation kinetics.

The representation of height versus oxidation time shows more strikingly the different growth rates for water and ethyl alcohol (Fig. 2). Oxide heights are measured with

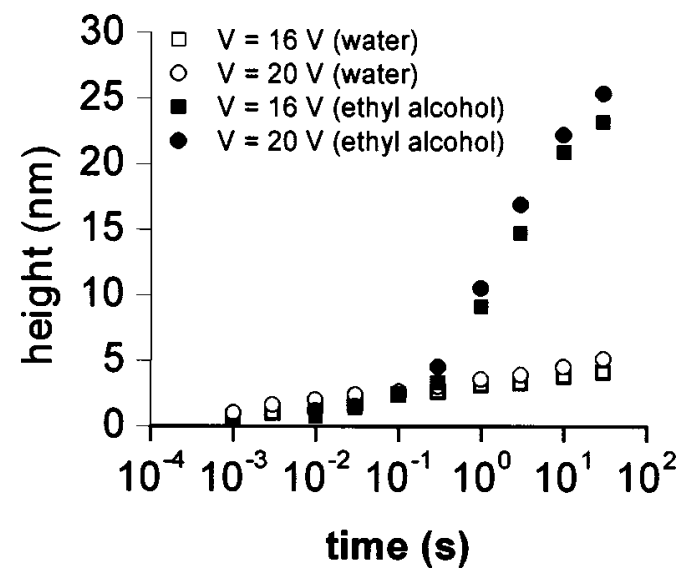

FIG. 2. Semilogarithmic dot height dependence on pulse duration and environment.

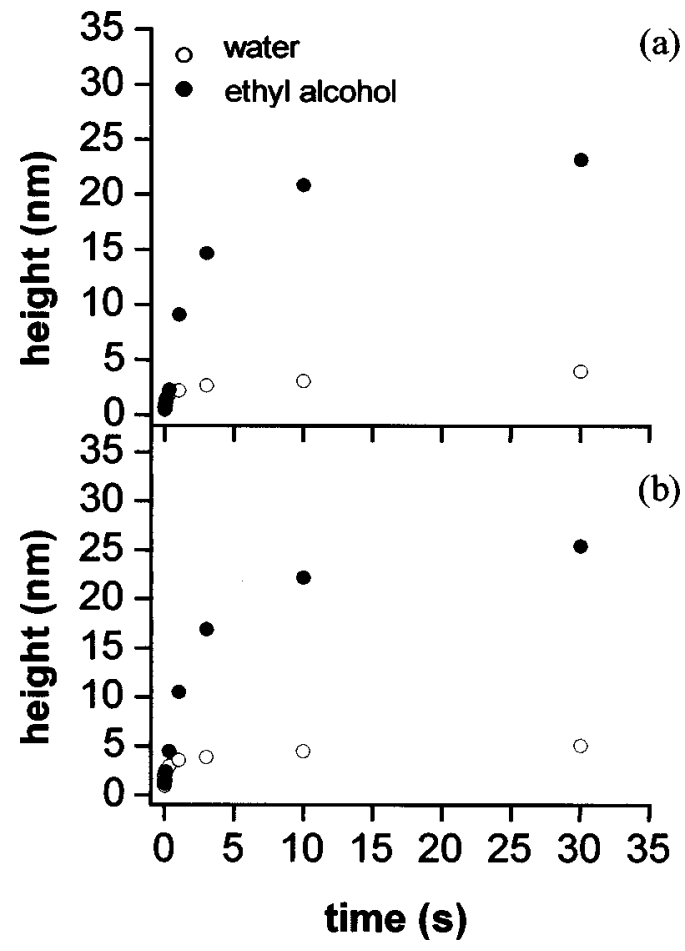

FIG. 3. Height of oxide dots vs oxidation time at different voltages: (a) 16 and (b) $20 \mathrm{~V}$.

respect to the base line defined by the silicon surface. The available data for local silicon oxides says that about $60 \%$ of the total oxide thickness lies above the silicon substrate base line. ${ }^{21}$ The semilogarithmic plot shows remarkable quantitative differences between oxides generated in aqueous and alcohol environments. At longer pulses, oxides in ethyl alcohol show height values above $20 \mathrm{~nm}$, i.e., 4-5 times higher than those obtained under identical conditions in water environments. The plot also shows that for short pulses, say below $0.01 \mathrm{~s}$ no oxides grow in ethyl alcohol environments, at least up to $24 \mathrm{~V}$, the maximum voltage applied here. Furthermore, while oxide heights in water show a linear dependence with $\log t / t_{c}$ this no longer applies for oxides grown in ethyl alcohol environments.

The linear plot of height versus time of Fig. 3 emphasizes two major results. First, it stresses the generality of the local oxidation process. With independence of the source of oxyanions, either water or alcohol the oxidation process shows similar qualitative trends. The kinetics is characterized by a rapid initial growth rate that slows down and selfterminates. However, the self-termination process is more dramatic in aqueous environments. Second, in ethyl alcohol is difficult or impossible to obtain oxides for pulse times below $0.01 \mathrm{~s}$ while they are easily generated in water. This indicates that either the generation of oxyanions in ethyl alcohol or their mobility limits the oxidation process. We conjecture that this is related to the different mobility of oxyanions $\left(\mathrm{OH}^{-}\right)$in water and ethyl alcohol. This is partially supported by observing that the viscosity of water at $20^{\circ} \mathrm{C}$ is smaller than that of ethanol (1.002 vs $1.2 \mathrm{cp})$. Then by assuming a linear regime, it can be deduced that the mobility of $\mathrm{OH}^{-}$in water is about 5 times higher than the mobility of $\mathrm{OH}^{-}$in ethanol $\left(20.6 \times 10^{-4} \mathrm{~cm}^{2} \mathrm{~V}^{-1} \mathrm{~s}^{-1}\right.$ vs 4.6

$\left.\times 10^{-4} \mathrm{~cm}^{2} \mathrm{~V}^{-1} \mathrm{~s}^{-1}\right)$. 


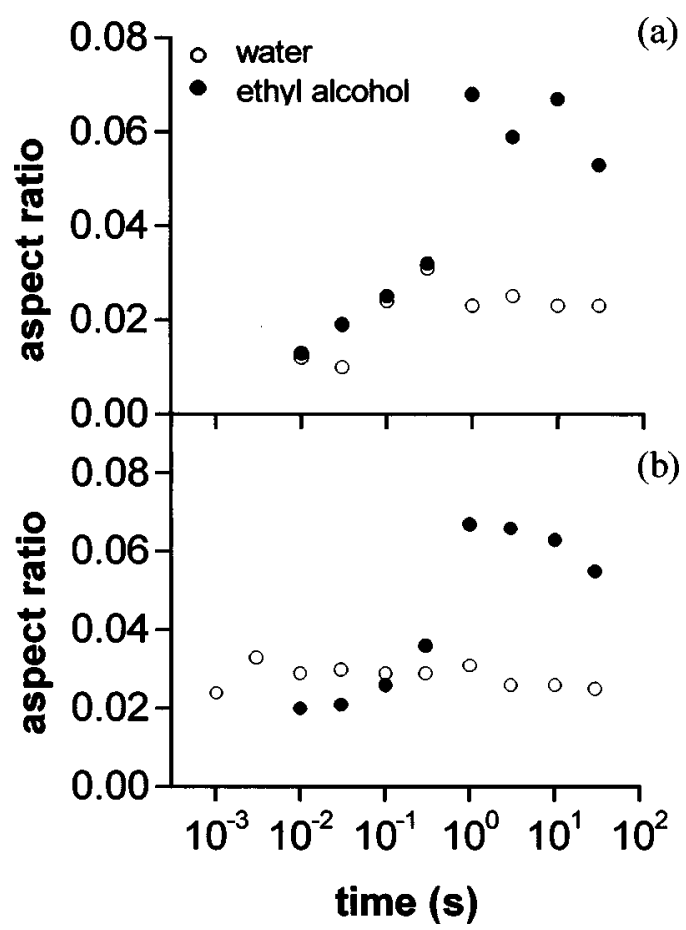

FIG. 4. Aspect ratio (height/width) dependence on pulse duration and environment: (a) $V=16 \mathrm{~V}$ and (b) $V=20 \mathrm{~V}$.

A considerable number of parameters are thought to be involved in the local oxidation process such as the electric field at the silicon oxide-silicon interface, the doping of the substrate, cantilever force constant, tip's chemical nature, and meniscus size and geometry. Consequently it has been hard to deduce analytical kinetics laws. For the earlier reason, several empirical kinetic expressions have been applied to fit the experimental data. ${ }^{11-14}$ Here we follow a power law parameterization, ${ }^{11,12}$

$$
h=b t^{\gamma},
$$

where $h$ is the oxide height about the substrate base line and $t$ is the duration of the pulse. The parameters $(b, \gamma)$ depend on the source of oxyanions and applied voltage. For example, at $20 \mathrm{~V}$ we have obtained $(9.3,0.32)$, and $(3.48,0.12)$ for ethyl alcohol and water, respectively, while for $V=16$ we have obtained $(8.62,0.32)$ and $(2.9,0.11)$, respectively. The parameter $b$ depends on both the applied voltage and the nature of liquid meniscus providing the oxyanions. On the other hand, $\gamma$ shows a strong dependence on the source of oxyanions. In all cases, the couple $(b, \gamma)$ shows higher numerical values in alcohol which implies a higher oxidation rate with respect to water. For long pulses, when spacecharge buildup controls the growth rate in water and a temporal crossover of the oxidation from transient to steady state is expected, ${ }^{12}$ a remarkable growth rate is observed in ethyl alcohol. Nonetheless the differences observed between oxidations in water and ethyl alcohol underline some of the hypothesis of the space-charge buildup model for aqueous environments. The presence of water molecules, hydrogen ions, and holes at the $\mathrm{Si} / \mathrm{SiO}_{2}$ interface favors the generation of positively charged defects of the type $\mathrm{H}_{3} \mathrm{O}^{+}{ }^{22}$ Those charges reduce the oxidation growth rate in two ways, first by capturing oxyanions and second by creating an electrical field that opposes the external field that drives the oxidation Downloaded 15 Sep 2003 to 161.111.235.247. Redistribution subj process. The absence of water molecules in ethyl alcohol prevents the Poindexter reaction to occur and consequently quenches, at least partially, the rapid decay of the oxidation growth.

The aspect ratio of the oxide dots (height/width) as a function of the oxidation time summarizes the earlier results (Fig. 4). For long pulses, say above $0.1 \mathrm{~s}$, oxidation in ethyl alcohol generates dots with aspect ratios above 0.06, i.e., two to three times higher than in water environments. However, for shorter pulses the aspect ratio is more favorable in water.

In short, we have extended local oxidation experiments to ethyl alcohol environments. Two major results have been found. First, the growth rate can be significantly increased by using oxyanions from ethyl alcohol molecules. We propose that the latter result is a consequence of the reduction of the trapped charges within the growing oxide. Second, the confirmation that local oxidation is a general process that only requires the presence of oxyanions and an electrical field to proceed the oxidation. The present results open the possibility of using local oxidation nanolithography to fabricate columnar structures while keeping lateral sizes in the nanometer range.

This work was supported by the European Commission (MONA-LISA, G5RD-2000-00349). The authors acknowledge stimulating discussions with Fernando Briones.

${ }^{1}$ E. S. Snow, P. M. Campbell, F. A. Buot, D. Park, C. R. K. Marrian, and R. Magno, Appl. Phys. Lett. 72, 3071 (1998).

${ }^{2}$ R. García, M. Calleja, and H. Rohrer, J. Appl. Phys. 86, 1898 (1999).

${ }^{3}$ E. B. Cooper, S. R. Manalis, H. Fang, H. Dai, K. Matsumoto, S. C. Minne, T. Hunt, and C. F. Quate, Appl. Phys. Lett. 75, 3566 (1999).

${ }^{4}$ K. Matsumoto, Y. Gotoh, T. Maeda, J. A. Dagata, and J. S. Harris, Appl. Phys. Lett. 76, 239 (2000).

${ }^{5}$ F. S. Chien, Y.-C. Chou, T. T. Chen, W.-F. Hsieh, T. S. Chao, and S. Gwo, J. Appl. Phys. 89, 2465 (2001).

${ }^{6}$ V. Bouchiat, M. Faucher, C. Thirion, W. Wernsdorfer, T. Fournier, and B. Pannetier, Appl. Phys. Lett. 79, 123 (2001).

${ }^{7}$ L. Pellegrino, I. Pallecchi, D. Marré, E. Bellingeri, and A. S. Siri, Appl. Phys. Lett. 81, 3849 (2002).

${ }^{8}$ T. Onuki, T. Tokizaki, Y. Watanabe, T. Tsuchiya, and T. Tani, Appl. Phys. Lett. 80, 4629 (2002).

${ }^{9}$ A. Dorn, M. Sigrist, A. Fuhrer, T. Ihn, T. Heinzel, K. Ensslin, W. Wegscheider, and M. Bichler, Appl. Phys. Lett. 80, 252 (2002).

${ }^{10}$ H. Sugimura and N. Nakagiri, Jpn. J. Appl. Phys., Part 1 34, 3406 (1995).

${ }^{11}$ T. Teuschler, K. Mahr, S. Miyazaki, M. Hundhausen, and L. Ley, Appl. Phys. Lett. 67, 3144 (1995).

${ }^{12}$ J. A. Dagata, F. Pérez-Murano, G. Abadal, K. Morimoto, T. Inoue, J. Itoh, and H. Yokoyama, Appl. Phys. Lett. 76, 2710 (2000); J. A. Dagata, T. Inoue, J. Itoh, K. Matsumoto, and H. Yokoyama, J. Appl. Phys. 84, 6891 (1998).

${ }^{13}$ E. Dubois and J.-L. Bubendorff, J. Appl. Phys. 87, 8148 (2000).

${ }^{14}$ M. Calleja and R. García, Appl. Phys. Lett. 76, 3427 (2000).

${ }^{15}$ M. Tello and R. García, Appl. Phys. Lett. 79, 424 (2001).

${ }^{16}$ M. Calleja, M. Tello, and R. García, J. Appl. Phys. 92, 5539 (2002); S. Gómez-Moñivas, J. J. Sáenz, M. Calleja, and R. García, Phys. Rev. Lett. 91, 056101 (2003).

${ }^{17}$ F. Marchi, V. Bouchiat, H. Dallaporta, V. Safarov, D. Tonneau, and P. Doppelt, J. Vac. Sci. Technol. B 16, 1 (1998).

${ }^{18}$ Y.-Y. Wei and G. Eres, Appl. Phys. Lett. 76, 194 (2000).

${ }^{19}$ R. García and A. San Paulo, Phys. Rev. B 61, R13381 (2000); A. San Paulo and R. García, ibid. 66, 041406(R) (2002).

${ }^{20} \mathrm{~V}$. Navarro and R. García (private communication).

${ }^{21}$ K. Morimoto, F. Pérez-Murano, and J. A. Dagata, Appl. Surf. Sci. 158, 205 (2000).

${ }^{22}$ E. H. Poindexter, C. F. Young, and G. J. Girardi, in Fundamental Aspects of Ultrathin Dielectrics on Si-Based Devices, edited by E. Garfunkel, E. Gusev, and A. Vul' (Kluwer, Dordrecht, 1998), p. 300.

to AIP license or copyright, see http://ojps.aip.org/aplo/aplcr.jsp 